\title{
International distribution and age estimation of the Portuguese BRCA2
}

\section{c.156_157insAlu founder mutation}

Ana Peixoto, Catarina Santos, Manuela Pinheiro, Pedro Pinto, Maria José Soares, Patrícia Rocha, Leonor Gusmão, António Amorim, Annemarie van der Hout, AnneMarie Gerdes, Mads Thomassen, Torben Kruse, Dorthe Cruger, Lone Sunde, Yves-Jean Bignon, Nancy Uhrhammer, Lucie Cornil, Etienne Rouleau, Rosette Lidereau, Koulis Yannoukakos, Drakoulis Yannoukakos, Maroulio Pertesi, Steven Narod, Robert Royer, Maurício M. Costa, Conxi Lazaro, Lidia Feliubadaló, Begoña Graña, Ignacio Blanco, Miguel de la Hoya, Trinidad Caldés, Philippe Maillet, Gaelle Benais-Pont, Bruno Pardo, Eitan Friedman, Eladio Velasco, Mercedes Durán, Maria-Dolores Miramar, Ana R. Valle, Ana Vega, Ana Blanco, Orland Diez, Teresa Ramon, Carmen Alonso, Montserrat Baiget, Judith Balmaña, William Foulkes, Marc Tischkowitz, Rachel Kyle, Nelly Sabbaghian, Patricia Ashton-Prolla, Ingrid P. Ewald, Thangarajan Rajkumar, Luisa M. Vieira, Giuseppe Giannini, Alberto Gulino, Maria I. Achatz, Dirce M. Carraro, Brigitte Bressac, Audrey Remenieras, Cindy Benson, Maureen Chung, Erik Teugels, Manuel R. Teixeira

Insert affiliations here:

Running title: Portuguese $B R C A 2$ founder mutation

Key words: c.156_157insAlu BRCA2 mutation, age estimation, hereditary breast/ovarian cancer.

Correspondence: Manuel R. Teixeira, MD, PhD, Department of Genetics, Portuguese Oncology Institute, Rua Dr. António Bernardino de Almeida, 4200-072 Porto, Portugal

Phone: +351 225084000; Fax: +351 225084016

E-mail: manuel.teixeira@ipoporto.min-saude.pt 


\section{Abstract}

The c.156_157insAlu BRCA2 mutation has so far only been reported in hereditary breast/ovarian cancer (HBOC) families of Portuguese origin. Since this mutation is not detectable using the commonly used screening methodologies and must be specifically sought, we screened for this rearrangement in a total of 5,440 suspected HBOC families from 22 labs from 13 countries from several continents. Whereas the c.156_157insAlu BRCA2 mutation was detected in 11 of 149 suspected HBOC families from Portugal, representing $37.9 \%$ of all deleterious mutations, in other countries it was detected only in one proband living in France and in four individuals requesting predictive testing living in France and in the USA, all having in common the fact that they are relatively recent immigrants of Portuguese origin in those countries. After performing an extensive haplotype study in carrier families, we estimate that this founder mutation has occurred 558 \pm 215 years ago. We further demonstrate significant quantitative differences regarding the production of the BRCA2 full length RNA and the transcript with exon 3 skipping in c.156_157insAlu BRCA2 mutation carriers and in controls, indicating that disruption of alternative transcript ratios is the mechanism causing hereditary breast/ovarian cancer associated with this BRCA2 rearrangement. We further show that the cumulative incidence of breast cancer in c.156_157insAlu BRCA2 mutation carriers does not differ from that of other BRCA2 and BRCA1 pathogenic mutations, further strengthening its role as the major contributor to hereditary predisposition to breast cancer in Portugal. We recommend that all suspected HBOC families from Portugal or with Portuguese ancestry are specifically tested for this rearrangement, ideally prior to screening of the entire coding regions of BRCA1 and BRCA2. 


\section{Introduction}

The pattern of BRCA1 and BRCA2 mutations in hereditary breast/ovarian cancer (HBOC) families varies widely among different populations. Many present a wide spectrum of different mutations throughout these genes, while some ethnic groups show a high frequency of particular mutations due to founder effects (Fackenthal and Olopade, 2007; Ferla et al., 2007). Identification of founder mutations in the various populations makes possible the use of more specific approaches to molecular testing (Filippini et al., 2007), allowing the analysis of more patients with less stringent selection criteria. Furthermore, a frequent founder mutation in a population allows a more accurate estimation of mutation-specific cumulative cancer incidence, facilitating also identification of genetic and environmental risk modifiers.

The c.156_157insAlu BRCA2 mutation was first described by Teugels et al. (2005) in a Portuguese patient residing in Belgium. These authors demonstrated that this exon 3 Alu insertion originates at the mRNA level an in-frame deletion of that exon, which encodes a transcriptional activation domain (Teugels et al., 2005). Machado et al. (2007) later described a regional founder effect for this rearrangement in HBOC families mostly originated from central/southern Portugal. We recently evaluated the contribution of the c.156_157insAlu BRCA2 mutation to inherited predisposition to breast/ovarian cancer in families originated mostly from northern/central Portugal (Peixoto et al., 2009a) and found that this rearrangement is responsible for more than half of all deleterious BRCA2 mutations and about one-fourth of all deleterious mutations in HBOC families. Additionally, in light of some doubts raised about the pathogenic effect of BRCA2 exon 3 skipping (Diez et al., 2007), we demonstrated its pathogenic effect by showing that the BRCA2 full length transcript is produced almost exclusively from the wildtype allele in patients carrying the c.156_157insAlu BRCA2 
rearrangement and that this mutation co-segregates with the disease in HBOC families and is absent in healthy blood donors, although residual exon 3 skipping in BRCA2 mRNA can be found in negative controls (Peixoto et al., 2009a; Peixoto et al., 2009b).

Although all reported c.156_157insAlu BRCA2 mutations have so far been identified in Portuguese HBOC families (Teugels et al., 2005; Machado et al., 2007; Peixoto et al., 2009a), this mutation is not detected using the commonly used screening methodologies and must be specifically sought (Teugels et al., 2005; Peixoto et al., 2009b), so one can not currently rule out its presence in other populations. To gain insight into the ancestral origin and population spread of the c.156_157insAlu BRCA2 mutation, we screened for this rearrangement in 5,440 suspected HBOC patients from several countries and performed an extensive haplotype study using closely linked microsatellite markers and single nucleotide polymorphisms (SNPs) in carrier families. In addition to estimate the age of the c.156_157insAlu BRCA2 mutation, we used realtime RT-PCR to quantify the preferential production of the transcript lacking exon 3 by the mutated allele and its (presumably residual) production by both alleles in noncarriers.

\section{Materials and methods}

\section{Families}

This study comprised a total of 5,440 suspected HBOC families from 22 labs from 13 countries from several continents. From Portugal, 149 new suspected HBOC families were selected for $B R C A 1$ and $B R C A 2$ mutation screening using previously described criteria (Peixoto et al., 2006; Peixoto et al., 2009a) after written informed consent. Molecular testing at the Department of Genetics of the Portuguese Oncology 
Institute, Porto, Portugal (IPO-Porto) started by looking for the c.156_157insAlu $B R C A 2$ mutation, followed by full BRCA1 and BRCA2 mutation screening with the previously reported methodology (Peixoto et al., 2006; Peixoto et al., 2009a; Peixoto et al., 2009b). The c.156_157insAlu BRCA2 mutation was additionally screened in 5,291 suspected HBOC families living in countries other than Portugal in whom no deleterious BRCA1/BRCA2 mutations had previously been found, with the following distribution: 1,209 from Spain (356 from Girona, 341 from Madrid, 151 from Valladolid, 132 from Zaragoza, 123 from Santiago de Compostela, and 106 from Barcelona), 1,087 from France (650 from Clermont-Ferrand, 428 from Saint-Cloud, and 9 from Villejuif, all the latter with Portuguese ancestry), 820 from Holland (Groningen), 758 from Denmark (Odense), 400 from Greece (Athens), 219 from Switzerland (Geneva), 200 from Belgium (Brussels), 185 from Israel (Tel Aviv), 144 from Brazil (98 from Porto Alegre and 46 from S. Paulo), 103 from Canada (Montreal), 91 from India (Chennai), and 75 from Italy (Rome). Besides the suspected HBOC families, two consecutive series of breast cancer patients from Rio de Janeiro, Brazil (390), and Ponta Delgada, Azores (86), were also screened for the c.156_157insAlu BRCA2 mutation. Additionally, predictive testing was performed in four individuals from two additional families (two relatives from each family living in Rhode Island, USA, and in Villejuif, France, respectively) with the c.156_157insAlu BRCA2 mutation identified elsewhere.

For the purpose of haplotype studies and age estimation of the c.156_157insAlu BRCA2 mutation, the 14 HBOC families we previously reported (Peixoto et al., 2009a) and the family (four c.156_157insAlu carriers) initially identified by Teugels et al. (2005) were also included. The geographic origin of the c.156_157insAlu BRCA2 positive families was inferred from the birthplace of the oldest carrier or of the oldest family member most likely to be a carrier. 


\section{Screening for the c.156_157insAlu BRCA2 mutation}

The detection of the c.156_157insAlu BRCA2 mutation was performed at the Department of Genetics of IPO-Porto for the suspected HBOC families from Portugal, for those originating from the Athens, Barcelona, Madrid and Zaragoza labs, as well as for predictive testing of four individuals from two additional families leaving in Rhode Island and Villejuif, respectively. The remaining cases were analyzed at the respective labs (except the cases from Rio de Janeiro, which were analyzed in Toronto) using the same protocol and a positive control provided by the Portuguese lab.

Screening for the c.156_157insAlu BRCA2 mutation was performed using two independent PCRs (Peixoto et al., 2009a; Peixoto et al., 2009b), one for exon 3 amplification and another specific for the Alu rearrangement. Using this strategy, we expect two bands in positive cases in the first PCR (one band if negative) and one band in the second PCR (none if negative). The second PCR helps to control the first PCR for eventual problems with preferential amplification of the shorter fragment (wild type), whereas the first PCR controls for eventual absence of amplification in the second PCR. This strategy of two independent PCRs, followed by sequencing of the genomic fragments in positive cases, allows the unambiguous detection of the c.156_157insAlu BRCA2 mutation (Peixoto et al., 2009a; Peixoto et al., 2009b). Positive and negative controls were used in all runs and all positive cases were confirmed in a second independent sample.

\section{Real-time RT-PCR analysis}

Primers and probes for the transcripts BRCA2 wild type (BRCA2-wt) and BRCA2 lacking exon 3 (BRCA2- $\triangle \mathrm{ex} 3)$ were designed with Primer Express 2.0 (Applied 
Biosystems, Foster City, USA). The forward primer for the BRCA2-wt transcript was TGAAATTTTTAAGACACGCTGCAA and the reverse primer was GGTTCGTAATTGTTGTTTTTATGTTCAG. The forward primer for the BRCA2- $\triangle \mathrm{ex} 3$ transcript was TCCAAAGAGAGGCCAACATTTT and the reverse primer was TTCACTGTGCGAAGACTTTTATGTC. The probes were 5'-FAMAAGCAGATTTAGGACCAATA-MGB-3' for the normal transcript (exon 2-3) and 5'FAM-AACAAAGCAGGAAGGAAT-MGB-3' for the BRCA2- $\triangle$ ex 3 transcript (exon 24). Primers and probes for the BRCA2 and GUSB (endogenous control) genes were purchased from Applied Biosystems. PCR reactions were performed in a $20 \mu \mathrm{l}$ volume reaction containing $9 \mu \mathrm{l}$ of synthesized cDNA, $10 \mu \mathrm{l}$ of TaqMan universal PCR master mix, $1 \mu \mathrm{M}$ of each primer and $0.25 \mu \mathrm{M}$ of probe. PCR was performed in separate wells for each primer/probe set and each sample was run in triplicate. PCR parameters were as follows: $50^{\circ} \mathrm{C}$ for $2 \mathrm{~min}, 95^{\circ} \mathrm{C}$ for $10 \mathrm{~min}$, followed by 50 cycles at $95^{\circ} \mathrm{C}$ for $15 \mathrm{~s}$ and $60^{\circ} \mathrm{C}$ for $1 \mathrm{~min}$. Each plate included multiple non-template controls and serial dilutions of a control to construct the standard curve of each transcript and endogenous control. To determine the relative expression levels of the target transcripts in each sample, the comparative $\mathrm{C}_{\mathrm{T}}$ method was performed as described by Schmittgen and Livak (2008) . The relative expression of the transcripts in two different groups (that included ten carriers and eight controls) was calculated using the $2^{-\Delta \mathrm{CT}}$ method. The ratio $2^{-}$ ${ }^{\Delta \mathrm{CT}} B R C A 2-\Delta \mathrm{ex} 3 / 2^{-\Delta \mathrm{CT}} B R C A 2-w t$ was calculated for each sample. The Mann-Whitney $\mathrm{U}$ Test was used to compare the relative expression of those transcripts between the two groups. Statistical analysis was performed with SPSS version 11 and statistical significance was considered whenever $P<0.05$.

\section{Mutation-specific cumulative incidence}


The cumulative incidence of breast cancer in women with the c.156_157insAlu BRCA2 mutation was derived using the method of Kaplan and Meier, with unaffected individuals censored at the age of last follow-up or death without breast cancer. Only individuals shown to be carriers or obligate carriers were used for this calculation.

\section{Microsatellite and SNP typing}

Haplotype analysis was carried out in families in which the c.156_157insAlu BRCA2 mutation was detected in at least one family member in addition to the proband. A total of 15 probands and 62 family members, including the three informative families previously reported (Peixoto et al., 2009a) and the one described by Teugels et al. (2005), were genotyped for polymorphic microsatellite markers flanking BRCA2 as described (Peixoto et al., 2009a). The D13S1699 marker was not included in this study because we could not rule out preferential allelic amplification. The physical distances of the genetic markers were derived from the National Center for Biotechnology Information (NCBI) Map Viewer $\quad$ (genome build 36.3) (http://www.ncbi.nlm.nih.gov/projects/mapview/). All nine markers were assayed by PCR using fluorescently 5'-labeled primers. PCR products were run on an ABI PRISM 310 Genetic Analyser (Applied Biosystems) together with the fluorescence labeled DNA fragment size standard TAMRA.

Single-nucleotide polymorphism (SNP) markers were used to obtain a haplotype spanning $\sim 1.1 \mathrm{Mb}$ encompassing the region between the D13S260 and D13S1695 microsatellite markers, where the first recombinant and/or mutational events were observed. In order to capture most of the genetic variation in this region and to avoid redundant SNP markers (i.e., markers in strong linkage disequilibrium), we performed Tag-SNP, namely Tagger Multimarker, using International HapMap Project CEPH 
(Utah residents with ancestry from northern and western Europe) population data (www.hapmap.org). We developed SNaPshot assays for 19 SNP markers by multiplexed nucleotide primer extension reaction using dye label terminators (Applied Biosystems). The primers for multiplex amplification and single base extension (Supplementary Table 1) were designed using the online Primer-BLAST tool (http://www.ncbi.nlm.nih.gov/tools/primer-blast/).

AutoDimer (www.cstl.nist.gov/strbase/NIJ/AutoDimer.htm) was used to test for potential hairpin structures and primer dimers. The 19 SNPs were PCR amplified in four multiplex reactions with amplicon length between 100 bp and 450 bp. Amplification was carried out in a 9700 Thermocycler (Applied Biosystems). After a $95^{\circ} \mathrm{C}$ pre-incubation step for 5 min, PCR was performed in a total of 35 cycles using the following conditions: $95^{\circ} \mathrm{C}$ denaturation for $30 \mathrm{~s}$, annealing at $55^{\circ} \mathrm{C}$ for $30 \mathrm{~s}$ and extension at $72^{\circ} \mathrm{C}$ during $30 \mathrm{~s}$, followed by 10 min of final extension at $72^{\circ} \mathrm{C}$. The multiplex SNaPshot reaction and capillary electrophoresis was done following the manufacturer's protocol (Applied Biosystems).

\section{Haplotype construction and estimation of mutation age}

Haplotype construction was performed manually based on the genotypes obtained of index cases and family members. We estimated the age of the c.156_157insAlu BRCA2 mutation from the variation accumulated in their ancestral haplotypes, as described by Martins et al. (2007). This method takes into account both recombination $(c)$ and mutation $(\mu)$ rates in the generation of variation. The probability of change per generation $(\varepsilon)$ is given by $\varepsilon=1-[(1-c)(1-\mu)]$, and the average of mutation and recombination events $(\lambda)$ equals $\varepsilon t$, where $t$ is the number of generations. The recombination rate $(c)$ was estimated from the physical distance between the two most 
distant markers (D13S1700 and D13S267) using a conversion factor calculated in Rutgers Map Interpolator (http://compgen.rutgers.edu/old/map-interpolator/). The estimate of average mutation rate used was $7.8 \times 10^{-4}$ (Gyapay et al., 1994) for dinucleotides and two times lower for tetranucleotides.

\section{Results}

\section{Detection of the c.156_157insAlu BRCA2 mutation}

Of the 149 Portuguese probands studied for germline mutations in the BRCA1 and BRCA2 genes at IPO-Porto, 11 patients presented the c.156_157insAlu BRCA2 mutation (Figure 1) and 18 patients presented other deleterious mutations in either BRCA1 (10 patients) or BRCA2 (8 patients) genes (data not shown). Together with the 14 probands we previously reported with this mutation (Peixoto et al., 2009a), a total number of 25 HBOC families have been uncovered at IPO-Porto at the time of writing. Altogether, 68 individuals from these 25 HBOC families have so far been tested for the c.156_157insAlu BRCA2 mutation and 39 of them were shown to be carriers. The known geographic origins of all the c.156_157insAlu BRCA2 positive families are shown in Supplementary Figure 1. Although most of the families are originated from northern/central Portugal, most likely reflecting our target population for genetic testing, we also detected the c.156_157insAlu mutation in families from southern Portugal and Madeira Island.

Of the 5,291 suspected HBOC families with no known deleterious mutation originated from other countries, only one proband tested in Clermont-Ferrand was shown to carry the c.156_157insAlu BRCA2 mutation. Interestingly, this patient belongs to a family of Portuguese origin living in France. Additionally, the two relatives living 
in Rhode Island (family with origin in Mangualde, central Portugal) and the two relatives living in Villejuif (family with origin in Porto, Portugal) for whom we performed predictive testing, were carriers of the c.156_157insAlu BRCA2 mutation that had previously been identified elsewhere in Portuguese family members. Finally, the patient originally reported by Teugels et al. (2005) belongs to a Portuguese family originally from the region of Guarda (central Portugal).

\section{Quantitative transcript analysis}

Real-time RT-PCR showed quantitative differences between the full length and the BRCA2- $\triangle \mathrm{ex} 3$ transcripts in c.156_157insAlu BRCA2 mutation carriers and controls. The relative expression of the $B R C A 2-\Delta \mathrm{ex} 3$ transcript was six fold higher in carriers compared with controls, whereas a three fold decrease was observed for the BRCA2-wt transcript in patients compared with controls (Figure 2). The difference observed between patients and controls was statistically significant $(P=0.00032)$.

\section{Mutation-specific cumulative incidence}

Using the method of Kaplan and Meier, the cumulative incidence of breast cancer in women carrying the c.156_157insAlu BRCA2 mutation was 90\% until the age of 60 years (Figure 3).

\section{Ancestral STR-based haplotypes and age estimate}

Nine different haplotypes were phased for 11 out of the 15 families, three of them reported earlier (Peixoto et al., 2009a). The results of the haplotype analyses for the 11 informative families are shown in Table 1 and the most parsimonious relationships among flanking haplotypes are presented as a phylogenetic network in 
Figure 4. The probability of mutation versus recombination was evaluated, considering the minimum number of stepwise mutations. In the 11 informative families, SNP haplotypes were constructed in order to establish if a specific microsatellite was different from the consensus because of a recombination event rather than a mutation (Supplementary Figure 2).

Based on the mutation and recombination events observed in microsatellite haplotypes and assuming a generation time of 25 years, the age estimate for the c.156_157insAlu BRCA2 mutation is 558 \pm 215 years (Table 1 ).

\section{Discussion}

The c.156_157insAlu BRCA2 mutation has so far only been reported in HBOC families of Portuguese origin. We here show that this rearrangement accounts for $57.8 \%$ of the BRCA2 mutations and $37.9 \%$ of all deleterious mutations in HBOC families originating mostly from northern/central Portugal. This study confirms our and other earlier findings indicating that this is by far the most common BRCA mutation in Portuguese families with hereditary predisposition to breast/ovarian cancer, being detected in about $8 \%$ of all probands tested and presenting a nation-wide distribution (Machado et al., 2007; Peixoto et al., 2009a). This high frequency makes it costeffective to test specifically for this rearrangement prior to screening the entire coding regions of BRCA1 and BRCA2 in suspected HBOC families from Portugal or with Portuguese ancestry. Furthermore, complementing earlier data showing that the c.156_157insAlu BRCA2 mutation originates skipping of exon 3 (Teugels et al., 2005) and that residual exon 3 skipping in BRCA2 mRNA can be found in negative controls (Peixoto et al., 2009a; Peixoto et al., 2009b), we here demonstrate by real-time RT-PCR that carriers present significantly more $B R C A 2-\Delta \mathrm{ex} 3$ transcripts and much less full 
length transcripts than controls, indicating that disruption of alternative transcript ratios is the mechanism causing hereditary breast/ovarian cancer associated with this BRCA2 rearrangement. We further show that the cumulative incidence of breast cancer in c.156_157insAlu BRCA2 mutation carriers does not differ from that of other BRCA2 and BRCA1 pathogenic mutations in our population (data not shown) or elsewhere (Ford et al., 1998), further strengthening its role as the major contributor to hereditary predisposition to breast cancer in Portugal.

Since the c.156_157insAlu BRCA2 mutation had only been reported in HBOC families of Portuguese origin (Teugels et al., 2005; Machado et al., 2007; Peixoto et al., 2009a) and is not detectable with commonly used screening methodologies, one can not exclude that it is present in other populations until it is specifically sought. To further evaluate whether or not it constitutes a population-specific founder mutation, we screened for the c.156_157insAlu BRCA2 rearrangement outside Portugal in more 5,291 suspected HBOC families with no known deleterious BRCA1/BRCA2 mutations coming from several countries mainly from Europe, but also from Asia and North and South America. In addition to the family identified in Belgium by Teugels et al. (2005), we now detected this mutation in one proband living in France and in four individuals requesting predictive testing living in France and in the USA, all having in common the fact that they are relatively recent immigrants of Portuguese origin in those countries. Interestingly, c.156_157insAlu BRCA2 mutation was not detected in 1.209 suspected HBOC families from Spain, including those from Galicia, the Spanish region with which Portugal shares more linguistic and cultural links, as also demonstrated by our recent finding of a common ancestry for the Portuguese HBOC families presenting the R71G BRCA1 founder mutation of Galician origin (Santos et al., 2009). 
The findings we here present indicate that, within the relatively large sample population studied, the c.156_157insAlu BRCA2 mutation is unique to HBOC of Portuguese ancestry, a fact that is hardly compatible with the age of about 2500 years previously estimated by Machado et al. (2007). Although geographic distribution of mutations is only an indirect measure of mutation age, more widespread mutations tend to be older than mutations showing a regional distribution. In order to get a more accurate mutation age estimate of the c.156_157insAlu BRCA2 rearrangement, we performed an extensive haplotype analysis having in mind that the size of an ancestral haplotype around a mutation is inversely correlated with the number of generations separating the common ancestor from the families carrying that rearrangement. After performing the haplotype reconstruction in the 11 informative families and assuming a generation time of 25 years, we estimate the age of the c.156_157insAlu BRCA2 mutation to be $558 \pm 215$ years, that is, most likely well after Portugal became politically independent (in 1143). Our estimate is consistent with the widespread distribution of the mutation in Portugal (Machado et al., 2007; Peixoto et al., 2009a; present report), the country demographic history (the North has been and still is consistently the source of migrants to the South), its occasional finding in countries with strong Portuguese immigration, and with its absence in the other populations studied (e.g. absence of the mutation in Spain, namely in Galicia). Nevertheless, statistical methods for estimating mutation ages are relatively crude (Rannala and Bertorelle, 2001), are dependent on sample representativeness, and estimate only the age of the common ancestor to the informative families that have been identified. The older age estimate advanced by Machado et al. (2007) was based upon a different sample of Portuguese patients (mostly from Center and South) and using a different age estimate method. However these authors recognize that the age of the mutation may be «overestimated, either because of the fact that mutation rates of the microsatellite markers were not taken into account or because recombination events in two 
families were considered». On the other hand, although the mutation has so far only been detected in Portugal and in a few families with Portuguese ancestry living in Belgium, France or the USA, we can not conclusively exclude its presence in other countries that have strong historical links with Portugal, such as those having Portuguese as official language (Brazil, Angola, Mozambique, Cape Verde, Guinea-Bissau, São Tomé and Príncipe, East Timor, and Macau) or other countries with a large community of Portuguese immigrants. In fact, one of our probands with the c.156_157insAlu BRCA2 mutation illustrates well this possibility: although she is now living in Portugal, her ancestors originating from North Portugal had moved several generations ago to Brazil and later to Angola, where reportedly various affected relatives lived.

In conclusion, we showed that the c.156_157insAlu BRCA2 rearrangement is a Portuguese founder mutation originated about $558 \pm 215$ years ago, accounting for the majority of the BRCA2 mutations and for about one third of all deleterious germline mutations in Portuguese HBOC families. We therefore recommend that all suspected HBOC families from Portugal or with Portuguese ancestry are specifically tested for this rearrangement, ideally prior to screening the entire coding regions of BRCA1 and $B R C A 2$. Furthermore, we showed that disruption of alternative transcript ratios is the mechanism causing hereditary breast/ovarian cancer associated with this BRCA2 rearrangement and that the cumulative incidence of breast cancer in c.156_157insAlu BRCA2 mutation carriers does not differ from that of other BRCA2 and BRCA1 pathogenic mutations.

\section{Acknowledgements}


This study was supported by Ministério da Saúde (Project No 15/2007). IPATIMUP was funded by Fundação para a Ciência e Tecnologia, through POCI (Programa Operacional Ciência e Inovação 2010). 


\section{References}

Diez, O., Gutierrez-Enriquez, S., Ramon y Cajal, T., Alonso, C., Balmana, J., and Llort, G. (2007). Caution should be used when interpreting alterations affecting the exon 3 of the BRCA2 gene in breast/ovarian cancer families. J Clin Oncol 25, 5035-5036.

Fackenthal, J.D., and Olopade, O.I. (2007). Breast cancer risk associated with BRCA1 and BRCA2 in diverse populations. Nat Rev Cancer 7, 937-948.

Ferla, R., Calo, V., Cascio, S., Rinaldi, G., Badalamenti, G., Carreca, I., Surmacz, E., Colucci, G., Bazan, V., and Russo, A. (2007). Founder mutations in BRCA1 and BRCA2 genes. Ann Oncol 18 Suppl 6, vi93-98.

Filippini, S., Blanco, A., Fernandez-Marmiesse, A., Alvarez-Iglesias, V., Ruiz-Ponte, C., Carracedo, A., and Vega, A. (2007). Multiplex SNaPshot for detection of BRCA1/2 common mutations in Spanish and Spanish related breast/ovarian cancer families. BMC Med Genet 8, 40.

Ford, D., Easton, D.F., Stratton, M., Narod, S., Goldgar, D., Devilee, P., Bishop, D.T., Weber, B., Lenoir, G., Chang-Claude, J., Sobol, H., Teare, M.D., Struewing, J., Arason, A., Scherneck, S., Peto, J., Rebbeck, T.R., Tonin, P., Neuhausen, S., Barkardottir, R., Eyfjord, J., Lynch, H., Ponder, B.A., Gayther, S.A., Zelada-Hedman, M., et al. (1998). Genetic heterogeneity and penetrance analysis of the BRCA1 and BRCA2 genes in breast cancer families. The Breast Cancer Linkage Consortium. Am J Hum Genet 62, 676-689.

Gyapay, G., Morissette, J., Vignal, A., Dib, C., Fizames, C., Millasseau, P., Marc, S., Bernardi, G., Lathrop, M., and Weissenbach, J. (1994). The 1993-94 Genethon human genetic linkage map. Nat Genet, pp. 246-339.

Machado, P.M., Brandao, R.D., Cavaco, B.M., Eugenio, J., Bento, S., Nave, M., Rodrigues, P., Fernandes, A., and Vaz, F. (2007). Screening for a BRCA2 rearrangement in high-risk breast/ovarian cancer families: evidence for a founder effect and analysis of the associated phenotypes. J Clin Oncol 25, 2027-2034.

Martins, S., Calafell, F., Gaspar, C., Wong, V.C., Silveira, I., Nicholson, G.A., Brunt, E.R., Tranebjaerg, L., Stevanin, G., Hsieh, M., Soong, B.W., Loureiro, L., Durr, A., Tsuji, S., Watanabe, M., Jardim, L.B., Giunti, P., Riess, O., Ranum, L.P., Brice, A., Rouleau, G.A., Coutinho, P., Amorim, A., and Sequeiros, J. (2007). Asian origin for the worldwide-spread mutational event in Machado-Joseph disease. Arch Neurol 64, 15021508.

Peixoto, A., Salgueiro, N., Santos, C., Varzim, G., Rocha, P., Soares, M.J., Pereira, D., Rodrigues, H., Bento, M.J., Fraguas, A., Moura, G., Regateiro, F., Castedo, S., and Teixeira, M.R. (2006). BRCA1 and BRCA2 germline mutational spectrum and evidence for genetic anticipation in Portuguese breast/ovarian cancer families. Fam Cancer 5, 379-387.

Peixoto, A., Santos, C., Rocha, P., Pinheiro, M., Principe, S., Pereira, D., Rodrigues, H., Castro, F., Abreu, J., Gusmao, L., Amorim, A., and Teixeira, M.R. (2009a). The 
c.156_157insAlu BRCA2 rearrangement accounts for more than one-fourth of deleterious BRCA mutations in northern/central Portugal. Breast Cancer Res Treat 114, 31-38.

Peixoto, A., Santos, C., Rocha, P., Pinto, P., Bizarro, S., and Teixeira, M.R. (2009b). Molecular diagnosis of the Portuguese founder mutation BRCA2 c.156_157insAlu. Breast Cancer Res Treat 117, 215-217.

Rannala, B., and Bertorelle, G. (2001). Using linked markers to infer the age of a mutation. Hum Mutat 18, 87-100.

Santos, C., Peixoto, A., Rocha, P., Vega, A., Soares, M.J., Cerveira, N., Bizarro, S., Pinheiro, M., Pereira, D., Rodrigues, H., Castro, F., Henrique, R., and Teixeira, M.R. (2009). Haplotype and quantitative transcript analyses of Portuguese breast/ovarian cancer families with the BRCA1 R71G founder mutation of Galician origin. Fam Cancer 8, 203-208.

Schmittgen, T.D., and Livak, K.J. (2008). Analyzing real-time PCR data by the comparative C(T) method. Nat Protoc 3, 1101-1108.

Teugels, E., De Brakeleer, S., Goelen, G., Lissens, W., Sermijn, E., and De Greve, J. (2005). De novo Alu element insertions targeted to a sequence common to the BRCA1 and BRCA2 genes. Hum Mutat 26, 284. 


\section{Figure legends}

Figure 1 - Molecular diagnosis of the BRCA2 c.156_157insAlu mutation using two independent PCR analyses, showing positive cases in lanes 1 and 2 and a negative case in lane 3. (A) PCR specific for BRCA2 exon 3, showing an additional band resulting from the insertion of a DNA fragment of about 350bp long within exon 3 of BRCA2 (c.156_157insAlu mutation; lane 1 and 2). (B) PCR specific for the c.156_157insAlu BRCA2 mutation with positive cases being then confirmed by sequencing the amplified genomic fragment. Lane 4 corresponds to a positive control and NTC is a non template control. MW refers to 100 bp DNA standard.

Figure 2 - Real-time RT-PCR quantification of the altered transcript ratios in BRCA2 c.156_157insAlu carriers as compared with controls. The relative expression of the BRCA2- $\triangle \mathrm{ex} 3$ transcript was six fold higher in carriers compared with controls, whereas a three fold decrease was observed for the BRCA2-wt transcript in patients compared with controls.

Figure 3 - Cumulative incidence of breast cancer among c.156_157insAlu BRCA2 germline mutation carriers, reaching $90 \%$ at about 60 years of age.

Figure 4 - Phylogenetic network showing the most parsimonious relationships among flanking short tandem repeat-based haplotypes in families carrying the c.156_157insAlu BRCA2 mutation. Circle and line sizes are proportional to the number of families and stepwise mutations, respectively. Dashed diamonds indicate recombination events. 
Supplementary Figure 1 - Map of Portugal showing the known geographical origin of the families with the c.156_157insAlu BRCA2 germline mutation. The black circles indicate the origin of the 25 families detected in Portugal (present report and those reported in Peixoto et al., 2009a), the open circle the origin of the family previously identified by Teugels et al. (2005) in Belgium, and the triangles the origin of the families of the four individuals (two from each) subjected to predictive testing living in Rhode Island, USA, and in Villejuif, France, respectively.

Supplementary Figure 2 - SNP marker haplotype spanning $\sim 1.1 \mathrm{Mb}$, encompassing the region between the D13S260 and D13S1695 microsatellite markers, in the 11 informative families. 
Table 1- Age estimation of the ancestral c.156_157insAlu BRCA2 mutation.

\begin{tabular}{|c|c|c|c|c|}
\hline Family ID & Haplotype $^{\mathrm{a}}$ & Families, No. & $\begin{array}{l}\text { Mutation steps/ } \\
\text { recombination events, } \\
\text { No. }\end{array}$ & $\operatorname{Age} \pm \delta, y^{b}$ \\
\hline $4001 ; 6216$ & H1: 317-160-156-299-230-242-228-144-158 & 2 & 0 & $558 \pm 215$ \\
\hline 5931 & H2: 317-160-156-295-230-242-228-144-158 & 1 & 1 & \\
\hline 6045 & НЗ: 313-160-156-299-230-242-228-144-158 & 1 & 1 & \\
\hline 14230 & H4: 269-160-156-299-230-242-228-144-158 & 1 & 1 & \\
\hline $7772 ; 14331$ & H5: 317-160-156-299-230-234-234-148-144 & 2 & 1 & \\
\hline 14459 & H6: 321-160-156-299-230-234-234-148-144 & 1 & 2 & \\
\hline 3854 & H7: 317-160-156-307-226-252-234-148-144 & 1 & 1 & \\
\hline 12703 & H8: 317-160-156-299-230-252-234-144-156 & 1 & 1 & \\
\hline 3286 & H9: 309-162-160-299-230-242-228-144-158 & 1 & 1 & \\
\hline Total & & 11 & 9 & \\
\hline $\begin{array}{l}\text { a The nine micr } \\
\text { D13S267 (from le } \\
\text { b The recombinat } \\
\text { conversion factor } \\
\text { seven dinucleotid }\end{array}$ & $\begin{array}{l}\text { ellite markers used were: D13S1700, D13S260, D1 } \\
\text { oright). Alleles conserved in each population appear in } \\
\text { rate }(c) \text { was based on the physical distance between th } \\
\text { culated in Rutgers Map Interpolator. The estimated pr } \\
\text { d two tetranucleotide short tandem repeats were studie }\end{array}$ & $\begin{array}{l}\text { 1698, D13S1701, } \\
\text { old. } \\
\text { two most distant } n \\
\text { ability of mutation }\end{array}$ & $\begin{array}{l}\text { 13S171, D13S1695, D13S1€ } \\
\text { kers (1930.8 kilobases; } c=0.0 \\
\text { ar generation and per haplotyl }\end{array}$ & $\begin{array}{l}\text { D13S310 } \\
97 \mathrm{cM} \text { ) usin } \\
\text { was } 0.00624\end{array}$ \\
\hline
\end{tabular}


1

2

3

4

NTC MW

3

4

NTC

A

B 


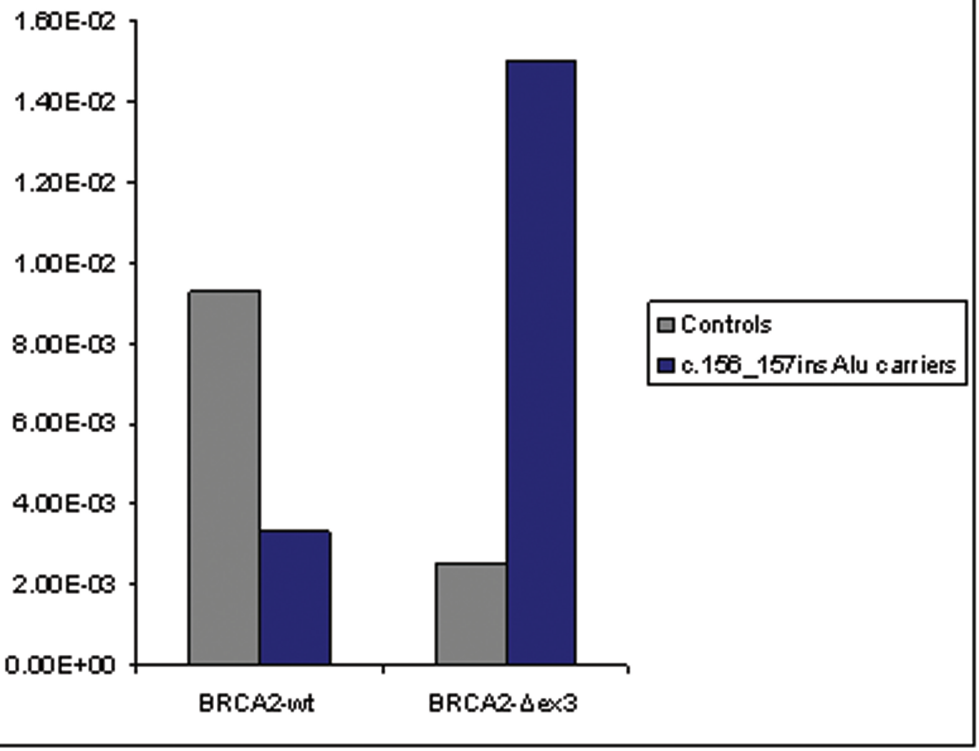




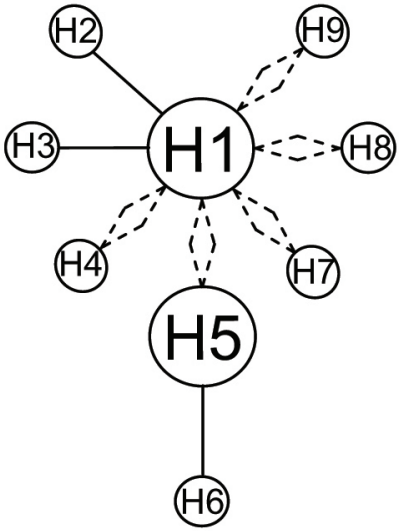




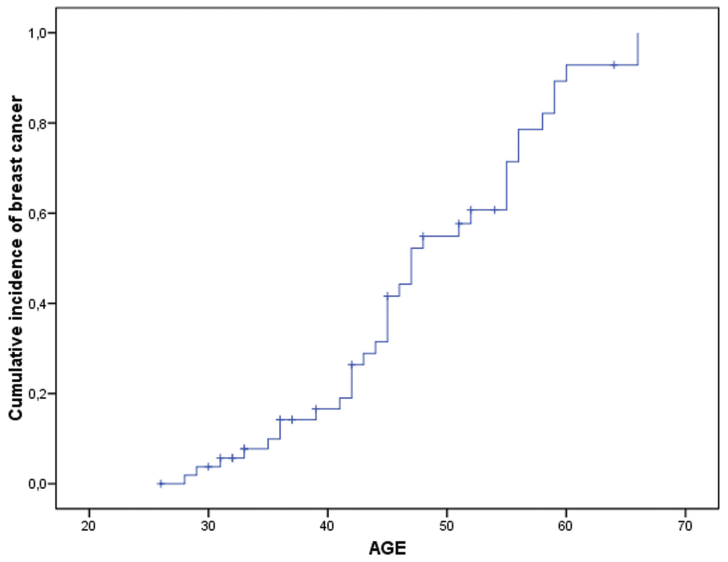


Supplementary table 1 - SNaPshot assay primers used for SNP genotyping around BRCA2.

\begin{tabular}{|c|c|c|c|c|}
\hline & SNP ID & PCR amplification primers & Single base extension primers & $\begin{array}{l}\text { Base } \\
\text { change }\end{array}$ \\
\hline \multirow[t]{10}{*}{1} & RS379693-F & ACCAGGCACGCATGTAATTC & ACAGTTGAAAAAGTACTTTCA & $\mathrm{A}>\mathrm{T}$ \\
\hline & RS379693-R & TGCCCAGCCAGAAACTTAAT & & \\
\hline & RS9590624-F & TTGGGAGGGAGAGCACTAGA & $(\mathbf{G A C T})_{2}$ GAAAGGTTATTGGAGACCTGC & $\mathrm{A}>\mathrm{T}$ \\
\hline & RS9590624-R & TGGGCTCCTACATCCAACTC & & \\
\hline & RS916732-F & TCACAGAGCTGTGCAAAACC & $(\text { GACT) })_{3}$ TAGAAAGTAAATGTTTGAAAATT & $\mathrm{C}>\mathrm{T}$ \\
\hline & RS916732-R & GCGGAATACCATCAACСАTC & & \\
\hline & RS2806638-F & TTCATCСАAСССТTCСАGTC & (GACT) $)_{5}$ ATTTTCTTCCTTTCTTTACAC & $A>G$ \\
\hline & RS2806638-R & AGCCACAGCAGTGAGAAAAT & & \\
\hline & RS206102-F & AAATGAAGCCTCTGTGACAAAA & (GACT) $)_{7}$ GTTTGCATCTTTTTTGAAGG & $\mathrm{C}>\mathrm{G}$ \\
\hline & RS206102-R & CCAGGGTGTCAGAGGAAAAA & & \\
\hline \multirow[t]{10}{*}{2} & RS9567445-F & GCTATGAGGCATAGTCAGCG & GGGAAACTCAGAAATTCAGT & $\mathrm{A}>\mathrm{T}$ \\
\hline & RS9567445-R & GCATTTGATGTGGTTAGCATTC & & \\
\hline & RS798972-F & ACGGCCACCATCTAACAGAC & GACTCAATTTCACCAAАATTTTACCA* & $\mathrm{A}>\mathrm{T}$ \\
\hline & RS798972-R & GCAGACGCСТССТСТСТСТА & & \\
\hline & RS703219-F & TTAGTGGCCCTGTTCTTGCT & $(\text { GACT) })_{3}$ GATAGTATTCCATTCCATGTCA* & $\mathrm{C}>\mathrm{T}$ \\
\hline & RS703219-R & AAAAACACCTGGCACAAAGC & & \\
\hline & RS582274-F & TCTCACCTGGCTGAAACTCC & $(\mathbf{G A C T})_{4}$ GATTGAAAATAGTTTGATCTCTTT & $\mathrm{C}>\mathrm{T}$ \\
\hline & RS582274-R & CTGCTTAGCAACAGGAAGGG & & \\
\hline & RS176059-F & AAGAAAAAGGGAGAAGTTTGCC & $(\text { GACT) })_{6}$ TTATATTTTGAGTCTAATTTGG & $A>G$ \\
\hline & RS176059-R & TAAACAAATCTGCCСССАТС & & \\
\hline
\end{tabular}


Supplementary table 1 (continued)

\begin{tabular}{|c|c|c|c|c|}
\hline Multiplex & SNP ID & PCR Amplification primers & Single base extension primers & $\begin{array}{l}\text { Base } \\
\text { change }\end{array}$ \\
\hline \multirow[t]{10}{*}{3} & RS1207952-F & TGCATGAAGCACACTGTGAA & GTTCAGCAGTTCTCTTTCAG & $A>C$ \\
\hline & RS1207952-R & CTCCTTGCTAGCCTCAGGG & & \\
\hline & RS2761367-F & AGCTGAGGATGGCTGAAAAA & GACTTCATTTTACTTTTTGTTTTGTTT* & $G>T$ \\
\hline & RS2761367-R & GACAAGCAGCCTAAGGGAAG & & \\
\hline & RS207632-F & GCAACGTTACAGAGCAGTTTG & $(\text { GACT })_{4}$ GAGCATCATGTCAATCAATG & $C>G$ \\
\hline & RS207632-R & ATGCTCCATCTCCACGTTTC & & \\
\hline & RS6561643-F & CCTCAGCAATCCTGTGTGTG & (GACT) $)_{5}$ GATGTTAGCTTGTTTTCCAAGT* & $\mathrm{A}>\mathrm{T}$ \\
\hline & RS6561643-F & GGGACTGGAGGATTTTCTCA & & \\
\hline & RS762900-F & GGCAAAAGGCAGTGCTAGTT & $(\text { GACT) })_{6}$ GACTGAATAGGCTTAAAATCTGAA* & $A>G$ \\
\hline & RS762900-R & TCTATACCAATGATGAGCAATCTT & & \\
\hline \multirow[t]{8}{*}{4} & RS9595946-F & TGCCTTTCCCACTCGTTAGT & TCTGAAAAGATTTGTGTTAATA* & $A>G$ \\
\hline & RS9595946-R & GGCTGAATTGAGTGATGGGT & & \\
\hline & RS472873-F & TTTCTGCATTGTGACTCTGCTT & $(\mathbf{G A C T})_{2}$ AACCAACTTCCTTCGTATTT & $\mathrm{T}>\mathrm{C}$ \\
\hline & RS472873-R & CACGGTAAAGGTTTCTGGGA & & \\
\hline & RS2146994-F & TCTCACCTGTGGGACCAAAT & (GACT) $)_{5}$ GATGGAGCTTTGGCCTCTTG & $A>C$ \\
\hline & RS2146994-R & TAGTGCTGTTCCTTGGGGAC & & \\
\hline & RS7983065-F & TGATGAGACCTGGTAGTCTGTAATG & (GACT) $)_{6}$ GAAAAAAATGATTTCCCTGAAA & $\mathrm{C}>\mathrm{T}$ \\
\hline & RS7983065-R & CCCTAGCAATTCTTCTGTATTTG & & \\
\hline
\end{tabular}





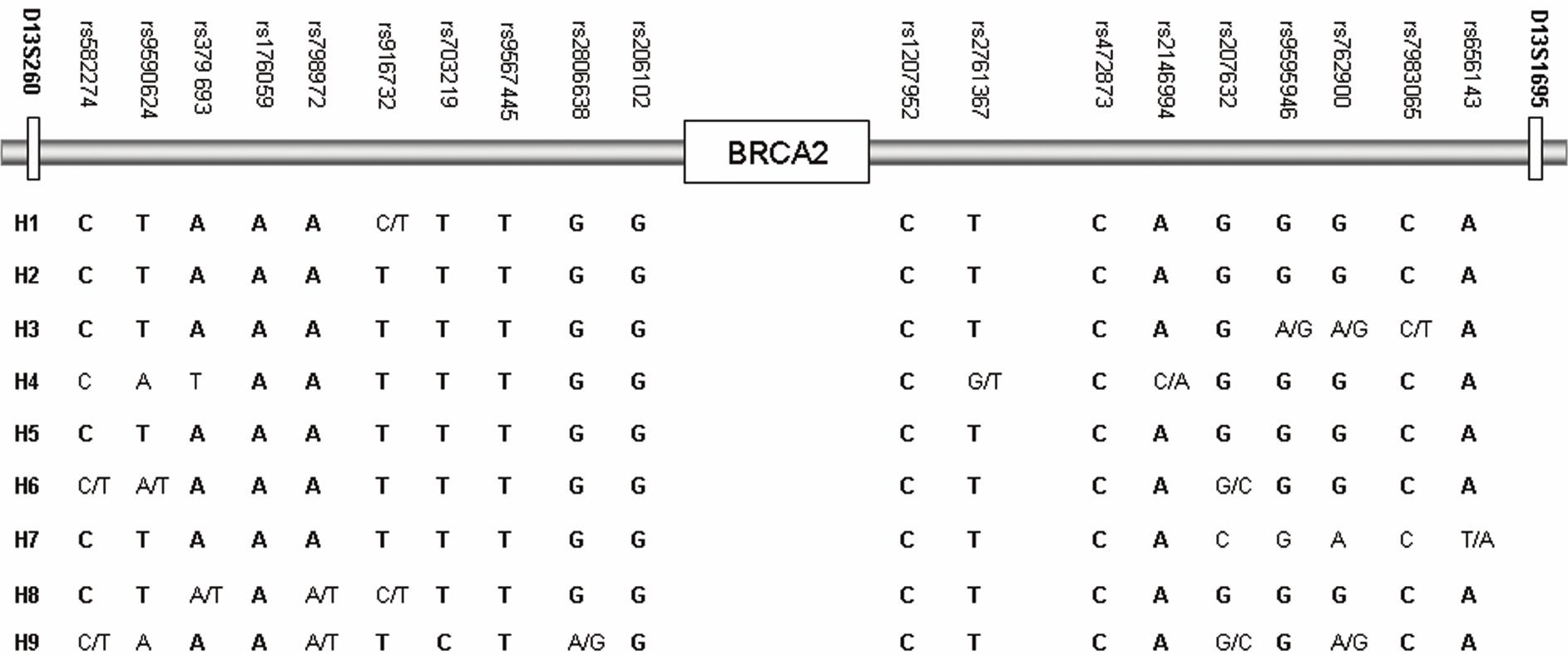

